\title{
Large-Signal DG-MOSFET Modelling for RFID Rectification
}

\author{
R. Rodríguez, ${ }^{1}$ B. González, ${ }^{1}$ J. García, ${ }^{1}$ A. Lázaro, ${ }^{2}$ B. Iñiguez, ${ }^{2}$ and A. Hernández ${ }^{1}$ \\ ${ }^{1}$ Institute for Applied Microelectronics, Universidad de Las Palmas de Gran Canaria, Edificio de Electrónica y Telecomunicación, \\ Campus Universitario de Tafira, 35017 Las Palmas, Spain \\ ${ }^{2}$ Departament d'Enginyeria Electrònica, Elèctrica i Automàtica, Universitat Rovira i Virgili, Escola Tècnica Superior d'Enginyeria, \\ Av. Dels Països Catalans 26, 43007 Tarragona, Spain
}

Correspondence should be addressed to B. González; benito@iuma.ulpgc.es

Received 6 September 2016; Accepted 4 October 2016

Academic Editor: Luis L. Bonilla

Copyright (C) 2016 R. Rodríguez et al. This is an open access article distributed under the Creative Commons Attribution License, which permits unrestricted use, distribution, and reproduction in any medium, provided the original work is properly cited.

\begin{abstract}
This paper analyses the undoped DG-MOSFETs capability for the operation of rectifiers for RFIDs and Wireless Power Transmission (WPT) at microwave frequencies. For this purpose, a large-signal compact model has been developed and implemented in VerilogA. The model has been numerically validated with a device simulator (Sentaurus). It is found that the number of stages to achieve the optimal rectifier performance is inferior to that required with conventional MOSFETs. In addition, the DC output voltage could be incremented with the use of appropriate mid-gap metals for the gate, as TiN. Minor impact of short channel effects (SCEs) on rectification is also pointed out.
\end{abstract}

\section{Introduction}

Nowadays, SOI technology offers wafers with thin and uniformly distributed oxide layers, exhibiting excellent electrical insulation and very high quality silicon/oxide interfaces. These properties encourage the design of different multiplegate devices [1-4] and development of compact models to account for their performance [5-7]. However, there is a lack of papers on applications with this type of transistors. The aim of this work, based on electrical simulations, is to demonstrate the feasibility of using Double Gate MOSFETs (DGMOSFETs) in rectifier circuits for RFIDs and Wireless Power Transmission (WPT) applications $[8,9]$ at microwave frequencies. The application of this technology for rectifier circuits has not yet been reported elsewhere, as far as we know.

The topology of the rectifier under consideration is shown in Figure 1, where two $n$-channel transistors, acting as charge pump, are connected [10]. The rectifier converts RF input to DC output power, as Figure 2 indicates. Gate and drain interconnections force transistors to operate in saturation or cut-off regimes. Thus, when the RF input signal, $V_{\mathrm{RF}}$, is in its negative half cycle, with $\left|V_{\mathrm{RF}}\right|>V_{\mathrm{th}}>0$ and $V_{\mathrm{th}}$ being the threshold voltage, the drain current of transistor DG$\mathrm{M} 1, I_{\mathrm{ds} 1}$, flows from ground to the coupling capacitor, with $C_{\mathrm{c}}=1 \mathrm{pF}$, and transistor DG-M2 remains off. Conversely, in the positive half cycle, with $V_{\mathrm{RF}}>V_{\text {th }}$, DG-M2 turns on, flowing its current, $I_{\mathrm{ds} 2}$, from the input terminal to the smoothing capacitor, with $C_{\mathrm{s}}=1 \mathrm{pF}$, with DG-M1 remaining off. In another case, both currents, $I_{\mathrm{ds} 1}$ and $I_{\mathrm{d} 22}$, are null. This process is repeated until the steady state is reached, when a DC output voltage is generated [10].

When DG-M2 switches off, $C_{s}$ can be discharged through load resistance, causing output ripples. In our study, we will assume a $10 \mathrm{k} \Omega$ output load to compare our results with those reported in [11].

The structure of this paper is as follows. In Section 2, the compact model implemented in the large-signal equivalent circuit is presented, including intrinsic capacitance for DGMOSFETs. Section 3 is devoted to analyse the rectifier performance, which is validated through numerical simulations with Sentaurus [12]. Technological and design aspects, as the use of different gate metal and number of stages, are also analysed in this section. Finally, some conclusions are exposed in Section 4. 


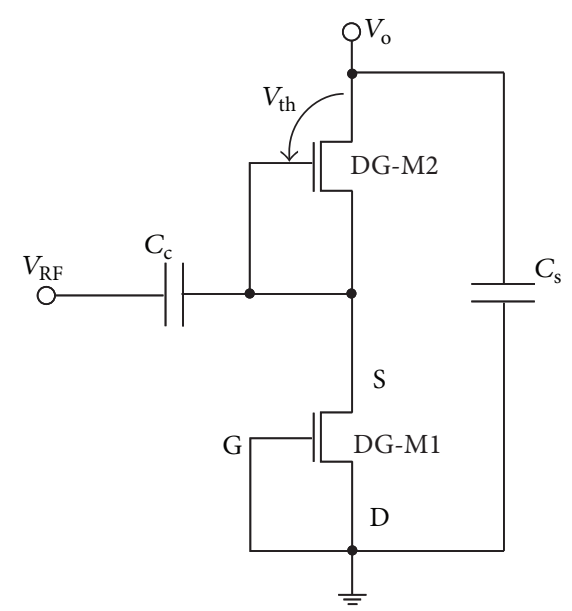

FIGURE 1: Schematic of the rectifier.

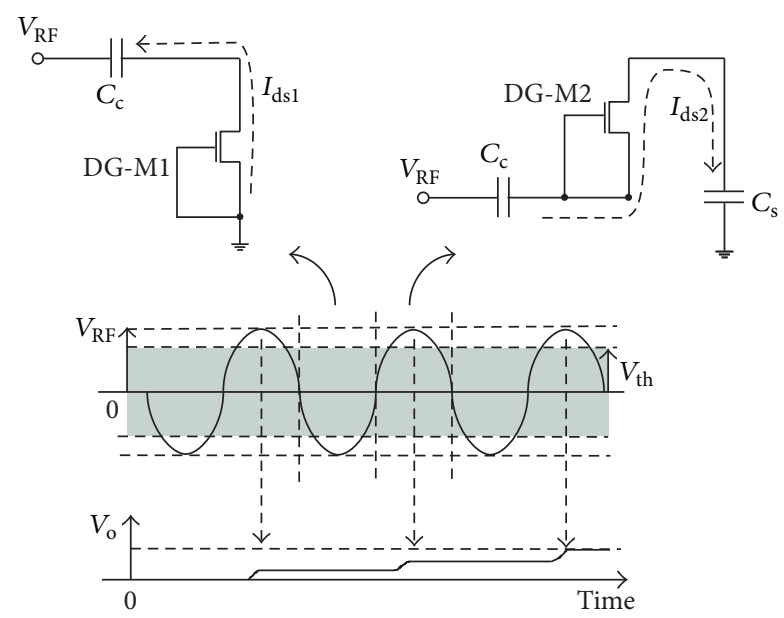

FIgURE 2: Rectification mechanism.

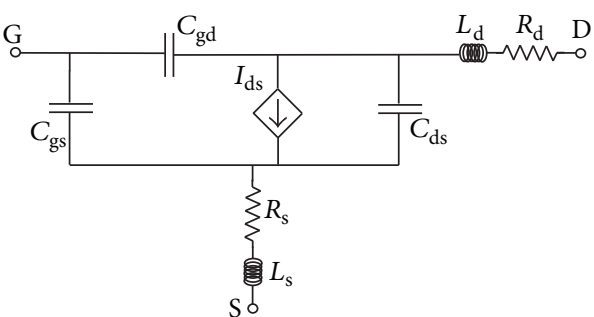

FIGURE 3: DG-MOSFET large-signal equivalent circuit.

\section{Large-Signal Equivalent Circuit}

The large-signal equivalent circuit proposed for the undoped DG-MOSFETs, with pads, is shown in Figure 3 [13]. It is composed by the intrinsic current, $I_{\mathrm{ds}}$, and capacitance from gate-to-source, $C_{g s}$, gate-to-drain, $C_{g d}$, and drain-tosource, $C_{\mathrm{ds}}$, and extrinsic source and drain resistances, $R_{\mathrm{s}}=$ $R_{\mathrm{d}}=0.32 \Omega$, and the extrinsic inductances, $L_{\mathrm{s}}=L_{\mathrm{d}}=$ $10^{-13} \mathrm{H}$.

The intrinsic current, based on the charge control model in [6], is given by

$$
\begin{aligned}
I_{\mathrm{ds}} & =\frac{W \mu}{L}\left[2 \beta\left(Q_{\mathrm{s}}-Q_{\mathrm{d}}\right)+\frac{Q_{\mathrm{s}}^{2}-Q_{\mathrm{d}}^{2}}{4 C_{\mathrm{ox}}}\right. \\
& \left.+8 \beta^{2} C_{\mathrm{Si}} \ln \left(\frac{Q_{\mathrm{d}}+2 Q_{\mathrm{o}}}{Q_{\mathrm{s}}+2 Q_{\mathrm{o}}}\right)\right],
\end{aligned}
$$

with the inversion charge, $Q$, being evaluated as

$$
Q=2 C_{\mathrm{ox}}\left\{-\frac{2 C_{\mathrm{ox}} \beta^{2}}{Q_{\mathrm{o}}}+\sqrt{\left(\frac{2 C_{\mathrm{ox}} \beta^{2}}{Q_{\mathrm{o}}}\right)^{2}+4 \beta^{2} \ln ^{2}\left[1+\exp \left(\frac{V_{\mathrm{gs}}-V_{\mathrm{th}}+\Delta V_{\mathrm{th}}-V}{2 \beta}\right)\right]}\right\},
$$

where $\beta$ is the thermal voltage, $Q_{\mathrm{o}}=4 \beta C_{\mathrm{Si}}\left(C_{\mathrm{Si}}=\right.$ $\left.\varepsilon_{\mathrm{Si}^{\mathrm{i}}} / t_{\mathrm{Si}}\right), C_{\mathrm{ox}}=\varepsilon_{\mathrm{SiO}_{2}} / t_{\mathrm{ox}}, Q_{\mathrm{s}}=Q(V=0)$, and $Q_{\mathrm{d}}=Q(V=$ $V_{\mathrm{ds}}$ ), with $V_{\text {th }}$ and $\Delta V_{\text {th }}$ (to assure the correct behaviour of $Q$ above threshold) as in [5].

For DG-M1 and DG-M2, a constant mobility, $\mu=$ $300 \mathrm{cmV}^{-1} \mathrm{~s}^{-1}$, is assumed, and the gate length, $L$, and gate width, $W$, are set to $0.18 \mu \mathrm{m}$ and $3.6 \mu \mathrm{m}$, respectively, with a gate oxide thickness of $t_{\mathrm{ox}}=2 \mathrm{~nm}, \mathrm{n}^{+}$polysilicon gate, and $t_{\mathrm{Si}}=20 \mathrm{~nm}$ for the thickness of the nonintentionally doped silicon layer.

Setting the gate-to-drain bias voltage, $V_{\text {gd }}$, the gate-tosource capacitance can be obtained as

$$
C_{\mathrm{gs}}=\left.\frac{d Q_{\mathrm{Tot}}}{d V_{\mathrm{gs}}}\right|_{V_{\mathrm{gd}}}
$$

$$
=\left.\left(\frac{d Q_{\mathrm{Tot}}}{d Q_{\mathrm{s}}}+\frac{d Q_{\mathrm{Tot}}}{d I_{\mathrm{ds}}} \cdot \frac{d I_{\mathrm{ds}}}{d Q_{\mathrm{s}}}\right) \cdot \frac{d Q_{\mathrm{s}}}{d V_{\mathrm{gs}}}\right|_{V_{\mathrm{gd}}},
$$

where $Q_{\text {Tot }}$ is the total channel charge in [6] and

$$
\begin{aligned}
\frac{d Q_{\mathrm{Tot}}}{d Q_{\mathrm{s}}} & =\frac{W^{2} \mu}{I_{\mathrm{ds}}} \cdot\left(\frac{Q_{\mathrm{s}}^{2}}{2 C_{\mathrm{ox}}}+\beta Q_{\mathrm{s}}+\frac{\beta Q_{\mathrm{s}}^{2}}{Q_{\mathrm{s}}+2 Q_{\mathrm{o}}}\right), \\
\frac{d I_{\mathrm{ds}}}{d Q_{\mathrm{s}}} & =\frac{W \mu}{L} \cdot\left(2 \beta+\frac{Q_{\mathrm{s}}}{2 C_{\mathrm{ox}}}-\frac{8 \beta^{2} C_{\mathrm{Si}}}{Q_{\mathrm{s}}+2 Q_{\mathrm{o}}}\right), \\
\frac{d Q_{\mathrm{s}}}{d V_{\mathrm{gs}}} & =\frac{1}{1 / 2 C_{\mathrm{ox}}+\beta\left(1 / Q_{\mathrm{s}}+1 /\left(Q_{\mathrm{s}}+2 Q_{\mathrm{o}}\right)\right)} .
\end{aligned}
$$


Substituting (4)-(6) in (3), with $d \mathrm{Q}_{\mathrm{Tot}} / d I_{\mathrm{ds}}=-\mathrm{Q}_{\mathrm{Tot}} / I_{\mathrm{ds}}$, $C_{\mathrm{gs}}$, finally results in

$$
C_{\mathrm{gs}}=\frac{\left(W^{2} \mu / I_{\mathrm{ds}}\right)\left[\left(Q_{\mathrm{s}}^{2} / 2 C_{\mathrm{ox}}+\beta Q_{\mathrm{s}}+\beta Q_{\mathrm{s}}^{2} /\left(Q_{\mathrm{s}}+2 Q_{\mathrm{o}}\right)\right)-Q_{\mathrm{Tot}} / W L \cdot\left(2 \beta+Q_{\mathrm{s}} / 2 C_{\mathrm{ox}}-8 \beta^{2} C_{\mathrm{Si}_{\mathrm{i}}} /\left(Q_{\mathrm{s}}+2 Q_{\mathrm{o}}\right)\right)\right]}{1 / 2 C_{\mathrm{ox}}+\beta\left(1 / Q_{\mathrm{s}}+1 /\left(Q_{\mathrm{s}}+2 Q_{\mathrm{o}}\right)\right)} .
$$

In a similar way, the drain-to-source capacitance can be written as

$$
\begin{aligned}
C_{\mathrm{ds}} & =-\left.\frac{d Q_{\mathrm{D}}}{d V_{\mathrm{ds}}}\right|_{V_{\mathrm{gd}}} \\
& =-\left.\left(\frac{d Q_{\mathrm{D}}}{d Q_{\mathrm{s}}}+\frac{d Q_{\mathrm{D}}}{d I_{\mathrm{ds}}} \cdot \frac{d I_{\mathrm{ds}}}{d Q_{\mathrm{s}}}\right) \cdot \frac{d Q_{\mathrm{s}}}{d V_{\mathrm{ds}}}\right|_{V_{\mathrm{gd}}},
\end{aligned}
$$

where $Q_{\mathrm{D}}$ is the total drain charge in [6]. Then, by replacing (5) in (8), with $d Q_{s} / d V_{\mathrm{ds}}=d Q_{\mathrm{s}} / d V_{\mathrm{gs}}$ (see (6)) and $d Q_{\mathrm{D}} / d I_{\mathrm{ds}}=-2 Q_{\mathrm{D}} / I_{\mathrm{ds}}, C_{\mathrm{ds}}$ is given by

$$
C_{\mathrm{ds}}=\frac{2 W \mu Q_{\mathrm{D}} / L I_{\mathrm{ds}} \cdot\left(2 \beta+Q_{\mathrm{s}} / 2 C_{\mathrm{ox}}-8 \beta^{2} C_{\mathrm{Si}} /\left(Q_{\mathrm{s}}+2 Q_{\mathrm{o}}\right)\right)-d Q_{\mathrm{D}} / d Q_{\mathrm{s}}}{1 / 2 C_{\mathrm{ox}}+\beta\left(1 / Q_{\mathrm{s}}+1 /\left(Q_{\mathrm{s}}+2 Q_{\mathrm{o}}\right)\right)},
$$

with

$$
\frac{d Q_{\mathrm{D}}}{d Q_{\mathrm{s}}}=\frac{\left(Q_{\mathrm{d}}^{3}-Q_{\mathrm{s}}^{3}\right) / \beta C_{\mathrm{ox}}+6\left(Q_{\mathrm{d}}^{2}-Q_{\mathrm{s}}^{2}\right)-12 Q_{\mathrm{o}}\left(Q_{\mathrm{d}}-Q_{\mathrm{s}}\right)+24 Q_{\mathrm{o}}^{2} \ln \left(\left(2 Q_{\mathrm{o}}+Q_{\mathrm{d}}\right) /\left(2 Q_{\mathrm{o}}+Q_{\mathrm{s}}\right)\right)}{12 C_{\mathrm{ox}} L I_{\mathrm{ds}}^{2}\left(2 Q_{\mathrm{o}}+Q_{\mathrm{s}}\right) / \beta W^{3} \mu^{2}\left[4 \beta C_{\mathrm{ox}}\left(Q_{\mathrm{o}}+Q_{s}\right)+2 Q_{\mathrm{o}} Q_{s}^{2}\right]}
$$

Finally, $C_{\mathrm{gd}}$ can be obtained by replacing $Q_{\mathrm{s}}$ by $Q_{\mathrm{d}}$ in (7), taking into account the DG-MOSFET symmetry. Therefore, $C_{\text {gd }}$ is given by

$$
C_{\mathrm{gd}}=\frac{\left(W^{2} \mu / I_{\mathrm{ds}}\right)\left[\left(Q_{\mathrm{d}}^{2} / 2 C_{\mathrm{ox}}+\beta Q_{\mathrm{d}}+\beta Q_{\mathrm{d}}^{2} /\left(Q_{\mathrm{d}}+2 Q_{\mathrm{o}}\right)\right)-Q_{\text {Tot }} / W L \cdot\left(2 \beta+Q_{\mathrm{d}} / 2 C_{\mathrm{ox}}-8 \beta^{2} C_{\mathrm{Si}} /\left(Q_{\mathrm{d}}+2 Q_{\mathrm{o}}\right)\right)\right]}{1 / 2 C_{\mathrm{ox}}+\beta\left(1 / Q_{\mathrm{d}}+1 /\left(Q_{\mathrm{d}}+2 Q_{\mathrm{o}}\right)\right)} .
$$

Note that all the capacitance and the drain current can be explicitly expressed in terms of the charge density in source and drain, $Q_{s}$ and $Q_{d}$.

\section{Rectifier Performance}

We implement the large-signal equivalent circuit for the DGMOSFET in Keysight Advanced Design System (ADS), using Verilog-A, which is the industry standard modelling language for analogic circuits [14].

In the rectifier under consideration (see Figure 1), gate and drain terminals in both DG-MOSFETs are shortcircuited. Thus, $C_{\mathrm{gd}}$ can be ignored, and $C_{\mathrm{gs}}$ and $C_{\mathrm{ds}}$ depend only on the drain-to-source voltage, $V_{\mathrm{ds}}$. Additionally, as Figure 4 shows, to avoid divergence problems by asymptotical response for $V_{\mathrm{ds}}$ close to zero, $C_{\mathrm{gs}}$ modelled capacitance and $C_{\mathrm{ds}}$ modelled capacitance (represented with symbols) are approximated by sigmoidal functions (represented with lines) as

$$
\left.C_{\xi \mathrm{d}}\right|_{V_{\mathrm{gd}}=0} \approx \frac{A_{0 \xi}}{1+e^{\left(A_{1 \xi}+A_{2 \xi} V_{\mathrm{ds}}\right)}}+A_{3 \xi}, \quad \text { with } \xi \equiv \mathrm{g}, \mathrm{d},
$$

with $A_{0 \xi}, A_{1 \xi}, A_{2 \xi}$, and $A_{3 \xi}$ being fitting parameters, summarised in Table 1 .

The electrical simulations for the rectifier in ADS are validated through numerical simulations with Sentaurus, accounting for the extrinsic elements of the transistors. Thus, Figures 5(a) and 5(b) show the dynamic drain current for DG-M1 and DG-M2, respectively, with RF input signal of $1 \mathrm{~V}$ amplitude at $1 \mathrm{GHz}$, after $10 \mathrm{~ns}$ (i.e., at steady values). It 
TABLE 1: Fitting parameters for $C_{\mathrm{gs}}$ and $C_{\mathrm{ds}}$.

\begin{tabular}{lccc}
\hline $\begin{array}{l}A_{0 \mathrm{~g}}, A_{0 \mathrm{~d}} \\
\left(\times 10^{-14} \mathrm{Fcm}^{-2}\right)\end{array}$ & $\begin{array}{c}A_{1 \mathrm{~g}}, A_{1 \mathrm{~d}} \\
-\end{array}$ & $\begin{array}{c}A_{2 \mathrm{~g}}, A_{2 \mathrm{~d}} \\
\left(\mathrm{~V}^{-1}\right)\end{array}$ & $\begin{array}{c}A_{3 \mathrm{~g}}, A_{3 \mathrm{~d}} \\
\left(\times 10^{-14} \mathrm{Fcm}^{-2}\right)\end{array}$ \\
\hline$-1.3,3.3$ & $-15.3,-4.9$ & $15.1,11.4$ & $1.3,-3.3$ \\
\hline
\end{tabular}

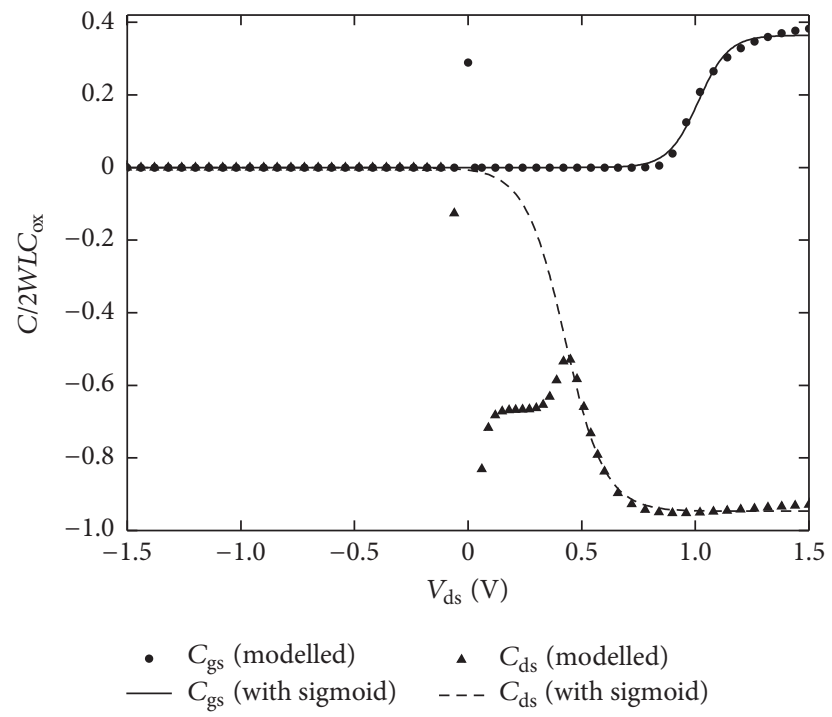

Figure 4: Modelled $C_{\mathrm{gs}}$ and $C_{\mathrm{ds}}$ (with symbols) and their sigmoidal approximations (with lines) versus drain-to-source voltage.

can be noticed that the numerical dynamic current (with symbols) is correctly modelled (with line) in both transistors. An unexpected negative modelled current for DG-M2, being off, appears, when ADS artificially adds auxiliary resistance, between gate and source, to facilitate convergence. However, its short duration, inferior to $0.1 \mathrm{~ns}$, makes DC output voltage deviations irrelevant.

Additionally, Figure 6 shows the numerical (with symbols) and modelled (with line) DC output voltage, varying the $\mathrm{RF}$ input power, at $5 \mathrm{GHz}$, from $-5 \mathrm{dBm}$ to $10 \mathrm{dBm}$. A good agreement between both data is achieved, with a maximum relative error of $11.7 \%$. Furthermore, the resulting power conversion efficiency (DC output - RF input power ratio) is similar to that reported in [10], with conventional MOSFETs.

Once the rectifier performance with ADS has been numerically validated, Figure 7 compares the transistor response for the electrical output voltage (with squares), for an input power of $5 \mathrm{dBm}$ at $5 \mathrm{GHz}$, with that for the rectifier implemented in HSPICE with commercial $0.18 \mu \mathrm{m}$ NMOS, from Texas Instruments, with identical dimensions (with solid line). Note that a similar DC output voltage of around $0.6 \mathrm{~V}$ is obtained, even when the threshold voltage for the NMOS is 0.18 volts lower than that for the DGMOSFET $(0.55 \mathrm{~V})$, which is compensated with its double current capability.

When threshold voltage is reduced, by using alternative gate metals, a higher rectified output voltage is expected. The use of titanium nitride (TiN) films as gate electrode in

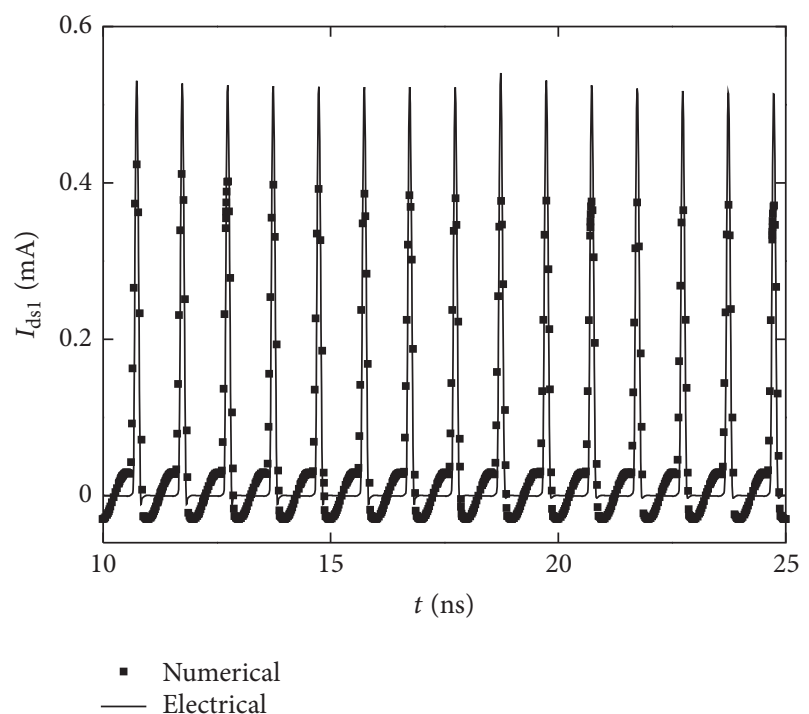

(a)

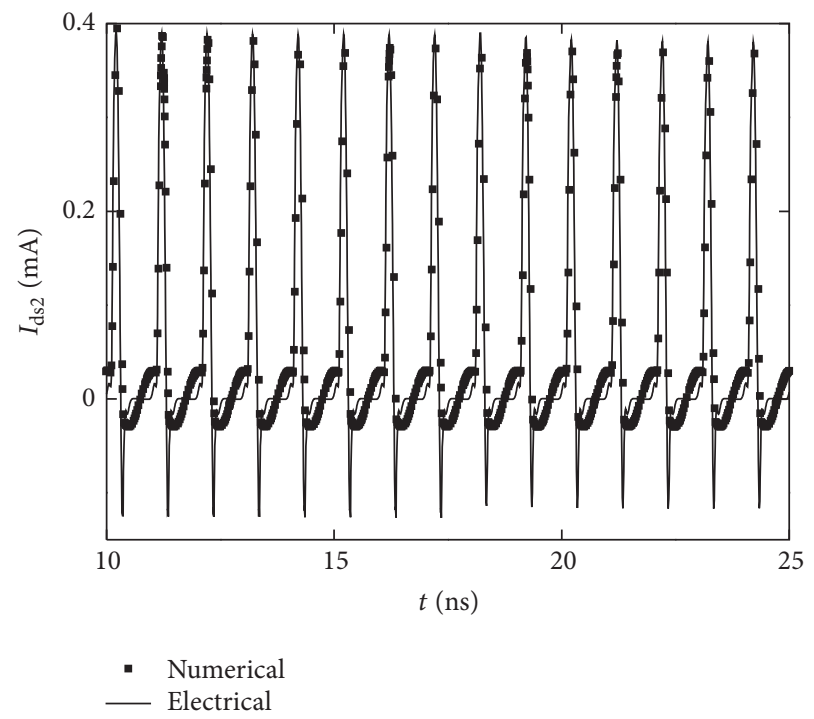

(b)

FIgURE 5: Numerical (with symbols) and modelled (with solid line) steady-state dynamic current for (a) DG-M1 and (b) DG-M2, with an input signal of $1 \mathrm{~V}$ amplitude at $1 \mathrm{GHz}$.

MOS capacitors and in Schottky diodes on n-type Si (100) substrates has been reported in [15], having a work function of $4.2 \mathrm{eV}$ and electrical resistivity of $270 \mu \Omega \mathrm{cm}$. Thus, when using TiN as the metal gate in the DG-MOSFETs, as Figure 7 indicates, the DC rectified voltage (with dotted line) grows $(0,25 \mathrm{~V})$ up to $0.83 \mathrm{~V}$.

On the other hand, the influence of the number of stages on the rectified output voltage has been analysed. In every stage (single rectifier in Figure 1), the source of DG-M2 must be connected to the drain of DG-M1 in next stage, with all coupling capacitance connected to the common RF input signal. Thus, the output voltage (with an output load of $10 \mathrm{k} \Omega$ ) is the sum of all voltages between the terminals of the smoothing capacitances, which are connected in series. 


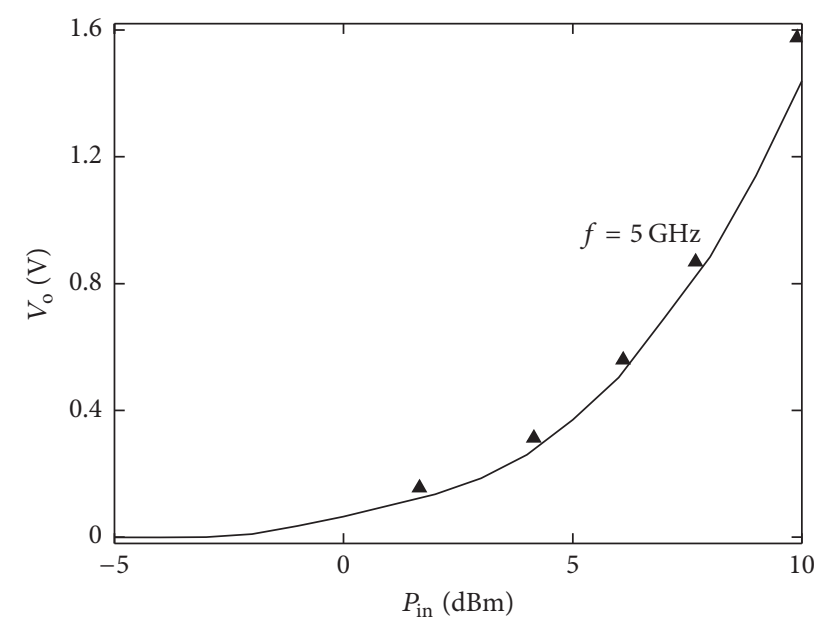

- Numerical

- Electrical

FIGURE 6: Numerical (with symbols) and modelled (with line) rectified output voltage as function of the RF input power at $5 \mathrm{GHz}$.

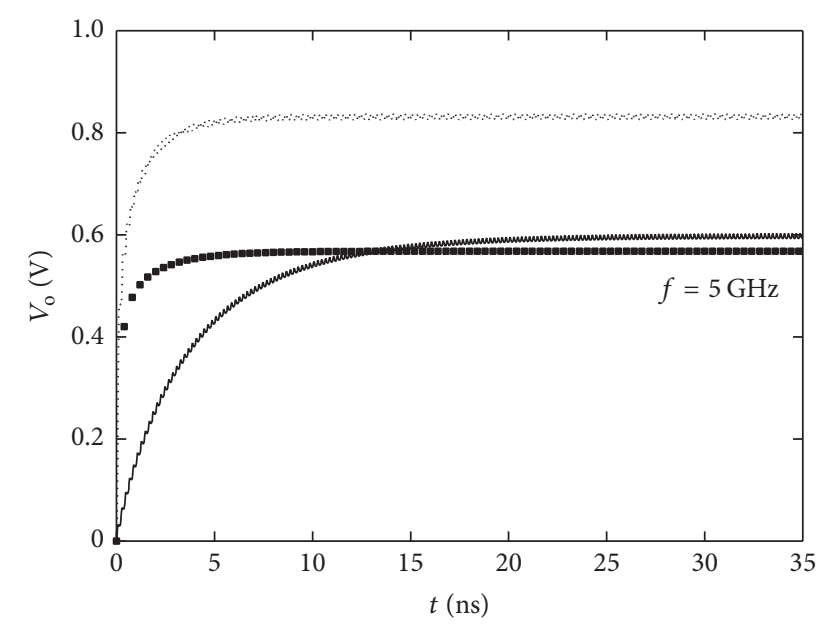

..... DG-MOSFET with TiN

- Conventional DG-MOSFET

_ Texas Instruments $0.18 \mu \mathrm{m}$-NMOS

FIgURE 7: Transient response for the rectified output voltage with conventional DG-MOSFETs (squares), Texas Instruments $0.18 \mu \mathrm{m}$ NMOS (solid line, with HSPICE), and DG-MOSFETs with TiN (dotted line), for an input power of $5 \mathrm{dBm}$ at $5 \mathrm{GHz}$.

Figure 8 shows the electrical output voltage when varying the $\mathrm{RF}$ input power from $-5 \mathrm{dBm}$ to $10 \mathrm{dBm}$, for different number of stages: from one to five, at $5 \mathrm{GHz}$ with $\mathrm{TiN}$ as gate metal. Note that, up to two stages, the output voltage increases, decreasing with additional stages: two is the optimal number of stages in the rectifier with DG-MOSFETs. This result differs from that reported in [10], with conventional N-MOSFETs, where six stages is the optimal configuration. Therefore, smaller number of stages than with conventional MOSFETs is necessary to produce the maximum output voltage with DG-MOSFETs.

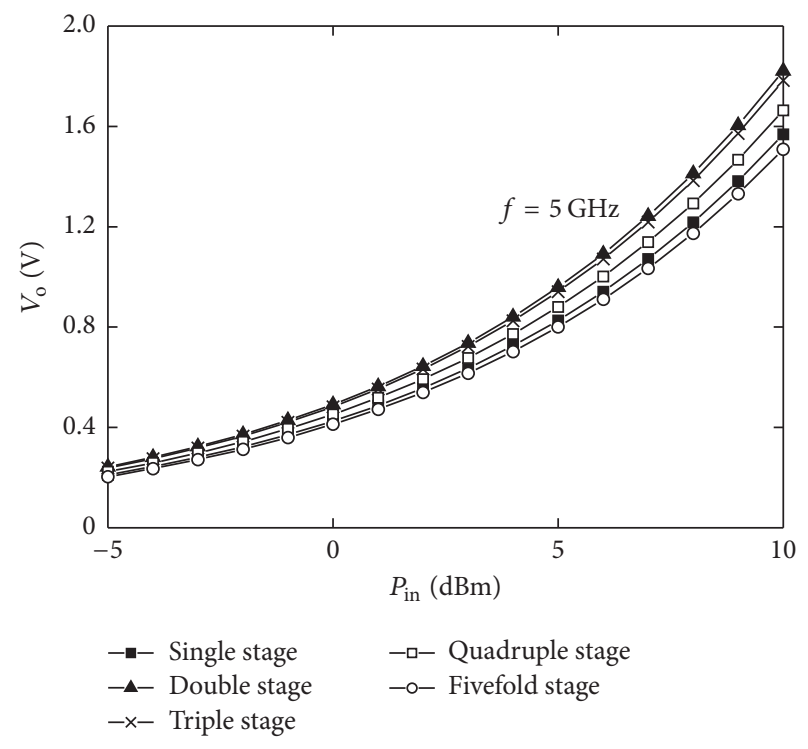

Figure 8: Modelled rectified output voltage for multiple stage configurations as function of the RF input power at $5 \mathrm{GHz}$ with TiN.

Finally, it can be pointed out that with short channel effects (SCEs) a superior current and, consequently, a higher DC output voltage would be expected. However, when incorporating the saturation velocity, effective mobility, and channel length modulation effects, as in [5], a minor impact on the DC output voltage is appreciated, which only increases $1.3 \%$ for an input power as high as $5 \mathrm{dBm}$ (at $5 \mathrm{GHz}$, with TiN), evidencing a determinant circuital topology influence.

\section{Conclusions}

A compact model for DG-MOSFETs has been developed and implemented in ADS, with Verilog-A, to perform electrical simulations of RFID rectifiers, which have been validated through numerical simulations with Sentaurus.

From transient simulations at microwave frequencies, it has been observed that the proposed rectifier with DGMOSFETs efficiently produces a DC output voltage. Furthermore, using TiN as metal gate, the DC output voltage increases $0.25 \mathrm{~V}$, compared with that obtained with $\mathrm{n}^{+}$ polysilicon. We have also demonstrated that just two stages are necessary to achieve the optimal performance of the rectifier, less than with conventional N-MOSFETs, and that SCEs have a minor impact on the DC output voltage.

\section{Competing Interests}

The authors declare that there are no competing interests regarding the publication of this paper.

\section{Acknowledgments}

This work has been supported by the Spanish national research project TEC2015-67883-R. 


\section{References}

[1] T. Mizuno, N. Sugiyama, T. Tezuka, T. Numata, and S.-I. Takagi, "High-performance strained-SOI CMOS devices using thin film SiGe-on-insulator technology," IEEE Transactions on Electron Devices, vol. 50, no. 4, pp. 988-994, 2003.

[2] S. K. Mohapatra, K. P. Pradhan, and P. K. Sahu, "Effect of channel \& gate engineering on Double Gate (DG) MOSFET-A Comparative Study," in Proceedings of the International Conference on Emerging Electronics (ICEE '12), pp. 1-3, December 2012.

[3] K.-H. Shih and C. O. Chui, "The low subthreshold swing possibility with asymmetries in double-gate SOI MOSFET," in Proceedings of the IEEE International SOI Conference, pp. 53-54, IEEE, New Paltz, NY, USA, October 2008.

[4] L. Wei, R. Zhang, K. Roy, Z. Chen, and D. B. Janes, "Vertically integrated SOI circuits for low-power and high-performance applications," IEEE Transactions on Very Large Scale Integration (VLSI) Systems, vol. 10, no. 3, pp. 351-362, 2002.

[5] B. Iñiguez, T. A. Fjeldly, A. Lázaro, F. Danneville, and M. J. Deen, "Compact-modeling solutions for nanoscale double-gate and gate-all-around MOSFETs," IEEE Transactions on Electron Devices, vol. 53, no. 9, pp. 2128-2142, 2006.

[6] O. Moldovan, D. Jiménez, J. R. Guitart, F. A. Chaves, and B. Iñiguez, "Explicit analytical charge and capacitance models of undoped double-gate MOSFETs," IEEE Transactions on Electron Devices, vol. 54, no. 7, pp. 1718-1724, 2007.

[7] D. E. Ward and R. W. Dutton, "A charge-oriented model for MOS transistor capacitances," IEEE Journal of Solid-State Circuits, vol. 13, no. 5, pp. 703-708, 1978.

[8] Y.-H. Suh and K. Chang, "A high-efficiency dual-frequency rectenna for $2.45-$ and $5.8-\mathrm{GHz}$ wireless power transmission," IEEE Transactions on Microwave Theory and Techniques, vol. 50, no. 7, pp. 1784-1789, 2002.

[9] J. Heikkinen and M. Kivikoski, "Low-profile circularly polarized rectifying antenna for wireless power transmission at $5.8 \mathrm{GHz}$," IEEE Microwave and Wireless Components Letters, vol. 14, no. 4, pp. 162-164, 2004.

[10] T. Umeda, H. Yoshida, S. Sekine, Y. Fujita, T. Suzuki, and S. Otaka, "A 950-MHz rectifier circuit for sensor network tags with 10-m distance," IEEE Journal of Solid-State Circuits, vol. 41, no. 1, pp. 35-41, 2006.

[11] K. Kotani, A. Sasaki, and T. Ito, "High-efficiency differentialdrive CMOS rectifier for UHF RFIDs," IEEE Journal of SolidState Circuits, vol. 44, no. 11, pp. 3011-3018, 2009.

[12] Sentaurus Device User's Guide, version I-2013, December 2013.

[13] N. B. Mihai, Compact modeling of the RF and noise behavior of multiple-gate MOSFETs [Ph.D. thesis], DEEAE, Universidad Rovira i Virgili, 2011.

[14] Agilent Technologies, ADS 2011, Verilog-A and Verilog-AMS Reference Manual, 2011.

[15] L. P. B. Lima, J. A. Diniz, I. Doi, and J. Godoy Fo, “Titanium nitride as electrode for MOS technology and Schottky diode: alternative extraction method of titanium nitride work function," Microelectronic Engineering, vol. 92, pp. 86-90, 2012. 

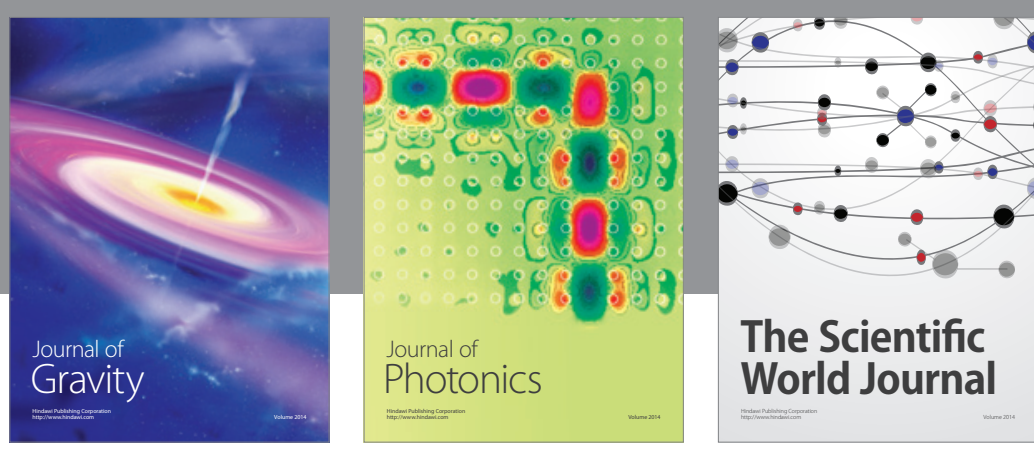

The Scientific World Journal
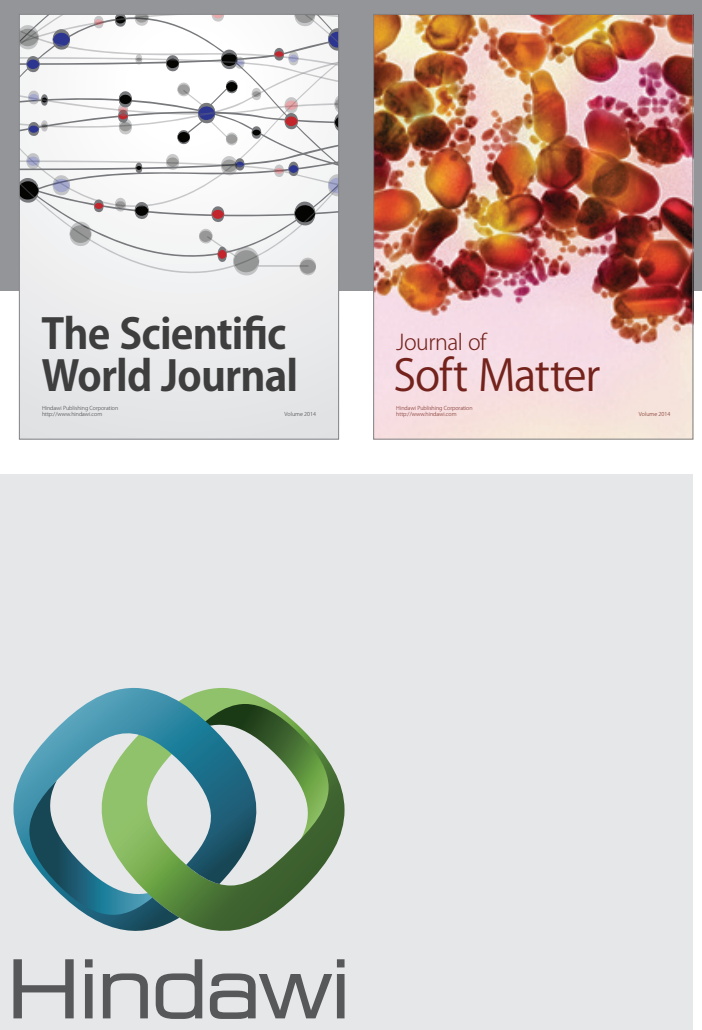

Submit your manuscripts at

http://www.hindawi.com

nternational Journal of

Statistical Mechanics
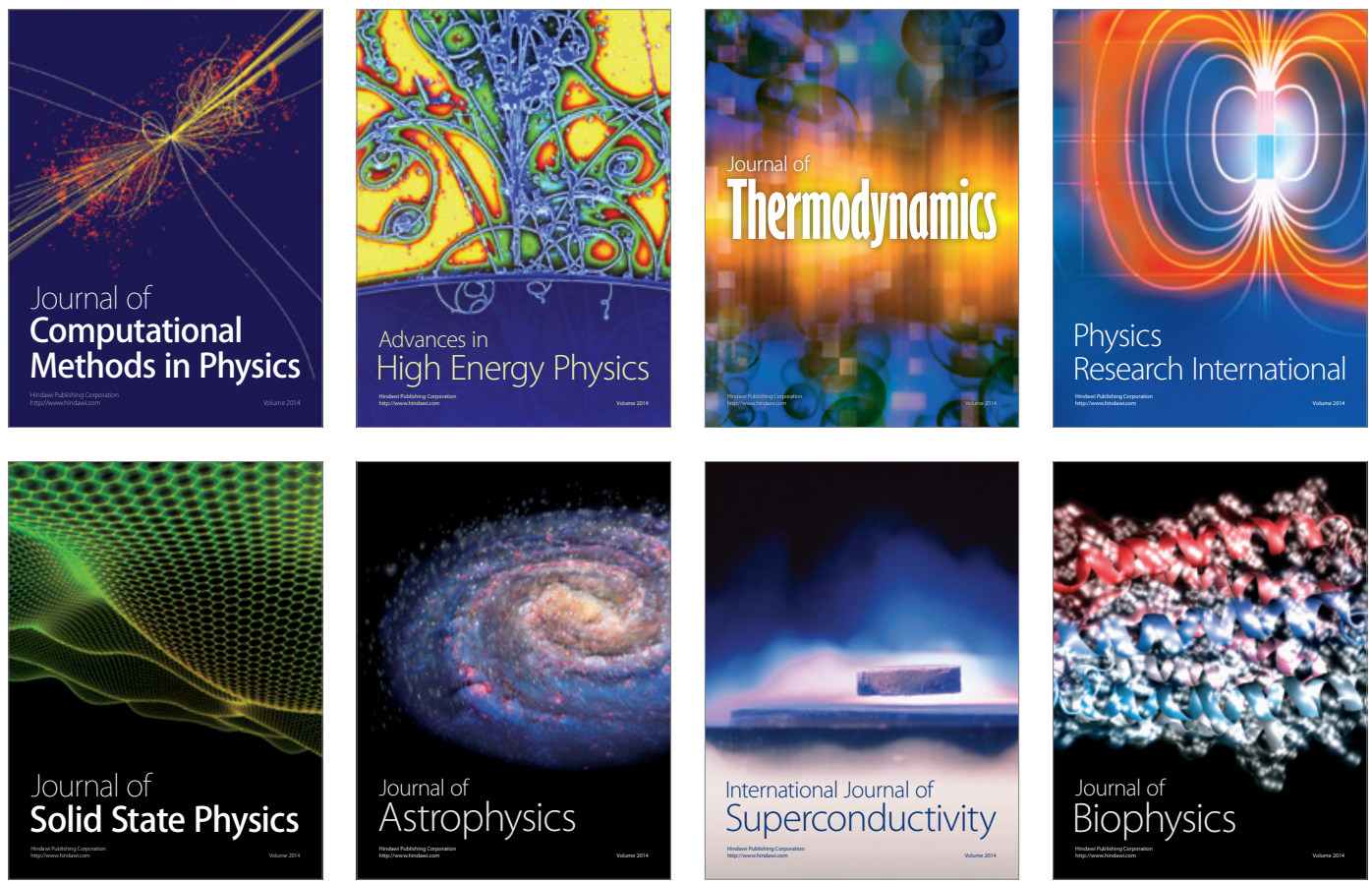
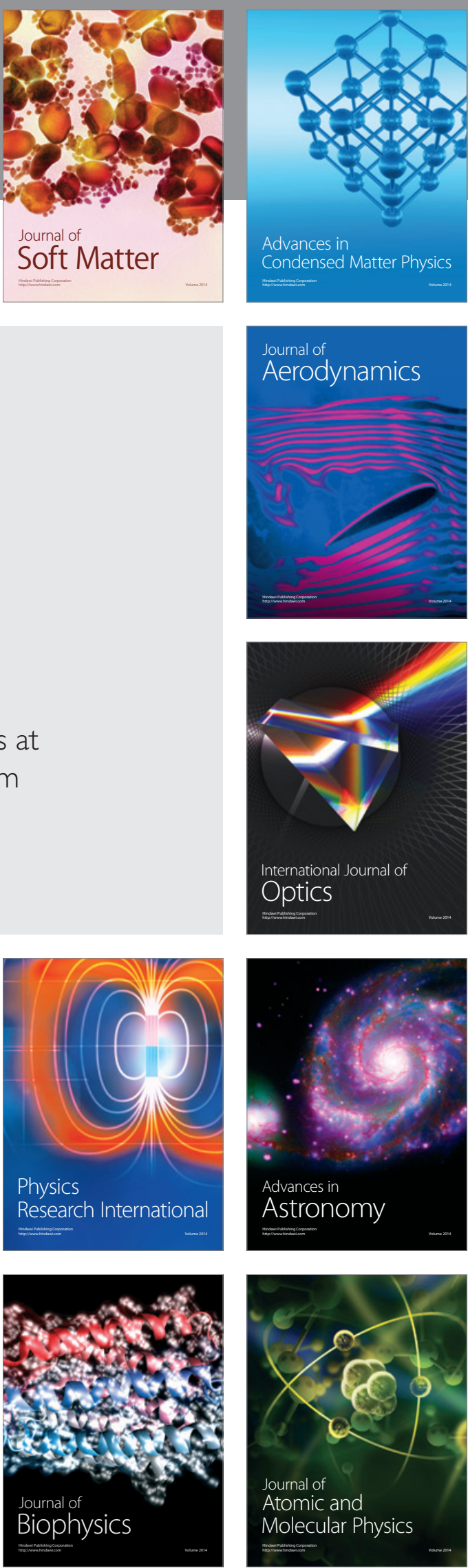